\title{
La psicomotricidad, un recurso para la mejora del grafismo en educación infantil \\ Psychomotor education, a resource for improving handwriting in infant education
}

\author{
Míriam Segura Meix ${ }^{1}$, Maria del Pilar Sabaté Curto² ${ }^{2}$ Cristóbal Caballé Barberá ${ }^{3}$ \\ miriam.segura@urv.cat, pilar.sabate@edificiseminari.com, cristobal.caballe@urv.cat
}

\author{
${ }^{1}$ Departamento de Pedagogia \\ Universidad Rovira y Virgili CTE
}

Tortosa, España

\author{
${ }^{2}$ Maestra de Educación Infantil \\ Colegio Sagrada Familia
}

Tortosa, España

\author{
${ }^{3}$ Psicomotricista \\ CPEE Sant Jordi (Diputación de \\ Tarragona) \\ Jesús-Tortosa, España
}

\begin{abstract}
Resumen- En esta investigación se analiza la relación grafismo psicomotricidad en alumnos de Educación Infantil. Estudiar como la psicomotricidad ayuda a mejorar la calidad del grafismo ha sido el objetivo del presente estudio. A partir de un diseño cuasi experimental, con medidas pre y pos-test con dos grupos, experimental y control, se analiza una muestra de 90 niños de cuatro años y se lleva a cabo la intervención a 45 de éstos. Se realizan análisis de comparación entre medias y medianas, de porcentajes de mejora y se utiliza la U de Mann Whitney como técnica de análisis de datos. Los resultados obtenidos nos indican cambios importantes en la calidad del grafismo en el grupo experimental (superiores a los 4, 4 puntos de media) tras haber practicado, durante doce sesiones, determinados ejercicios de psicomotricidad fina $y$ gruesa. Finalmente, los resultados confirman nuestra hipótesis y proponemos que el aprendizaje del grafismo, siguiendo las leyes del desarrollo motor, sea abordado desde una perspectiva global, que incluya tanto la ejercitación de la motricidad fina como de la gruesa.
\end{abstract}

Palabras clave: Educación Infantil, psicomotricidad fina, psicomotricidad gruesa, grafomotricidad, escritura.

Abstract- This study analyses the relation between handwriting and psychomotor education in pupils in Infant Education and how psychomotor education helps to improve the quality of handwriting. A quasi-experimental study design involving pre- and post-test measurements of an experimental group and a control group was used to analyse a sample of 90 four-year-old children. Of these, 45 were the subject of an intervention. Means, medians and improvement percentages are compared and analysed and the Mann-Whitney Utest is used to analyse the data. The results indicate significant changes in the quality of the experimental group's handwriting (a mean of over 4.4 points) after they had carried out twelve sessions of specific fine and gross psychomotor exercises. Finally, the results confirm our hypothesis and we propose that handwriting should be taught in line with motor development and from a global perspective that includes the exercising both fine and gross motor skills.

Keywords: Pre-school education, slim motor skills, thick motor skills, graphomotricity, writing, development laws.

\section{INTRODUCCIÓN}

El aprendizaje de la lectoescritura empieza ya en la primera infancia $\mathrm{y}$, mucho antes, aparece la necesidad del niño de representar sonidos, imágenes o ideas sobre el papel, la pizarra o la arena por ejemplo; es lo que conocemos como grafismo. De acuerdo con Sugrañes, Àngel, Andrés, Colomé, Martí, Martín, Pinell, et al. (2007, p. 283), el grafismo o actividad gráfica es "la producción de trazos sobre cualquier superficie a partir de un desplazamiento que se puede realizar con todo el cuerpo o con alguno de sus segmentos, utilizando o no un objeto o instrumento". Debido a la importancia de este proceso de aprendizaje y su significación para los aprendizajes posteriores, son muchos los estudios que relacionan la escritura en edad preescolar con las habilidades metalingüísticas (Albuquerque \& Alves-Martins, 2016), o con el conocimiento del sistema alfabético (Tolchinsky, 2005; Shatil, Share \& Levin, 2000). Otros autores como Fonseca (2008), Ajuriaguerra, Auzias, \& Denner (1973) y Portellano (1985), proponen la educación psicomotriz como instrumento de prevención de las dificultades de aprendizaje escolar y coinciden en la importancia del proceso de la escritura y su complejidad.

Alviz (2012, p.90), describe la grafomotricidad como "un acto motórico que comienza con la macromotricidad o motricidad gruesa (desplazamiento del cuerpo en el espacio), continúa con la motricidad media (movimiento del cuerpo y miembros, sin cambiar de lugar), y termina con la motricidad fina (manos y dedos, a la vez que dibuja aquel gran movimiento inicial)”. Es por lo tanto un proceso interno que requiere de una sólida base psicomotriz.

Diferentes autores ya clásicos, entre ellos Ajuriaguerra et al. (1973), Auzias (1978), Lurçat (1988) y Calmy (1977), hacen referencia a la gran complejidad del estudio de la grafomotricidad y plantean el grafismo como una actividad psicomotriz en la cual podemos distinguir cuatro ámbitos o niveles funcionales: el ámbito motor, el perceptivo, el representativo y el afectivo.

Siguiendo con Ajuriaguerra et al. (1973) los factores implicados en el desarrollo del grafismo son el ejercicio y el desarrollo motor, contemplando éste último la maduración general del sistema nervioso, el desarrollo psicomotor general y el desarrollo de la motricidad fina. Para Portellano (1989), este desarrollo motor del que hablamos sigue unas leyes fundamentales, que Coghill enunció en 1929, y son la ley de maduración céfalo caudal, que establece que el desarrollo 
procede desde la parte superior del cuerpo a la inferior y la ley próximo distal, que establece que el desarrollo de las partes más próximas al eje central del cuerpo maduran primero que las partes del cuerpo que están más lejos. A las anteriores leyes de desarrollo podemos añadir otras dos leyes, que las amplían y complementan: la ley de actividades en masa a las específicas (general-específico) y la ley de desarrollo de flexores y extensores.

La grafomotricidad es un proceso extremadamente complejo. El desarrollo motor es un factor esencial en el desarrollo de la escritura. Se trata de un acto neuro-perceptivomotor que requerirá capacidades psicomotoras de inhibición, control neuromuscular, coordinación óculo-manual y organización espacio temporal, además de una coordinación funcional de la mano, con independencia de la mano y los dedos en relación con el brazo, así como la coordinación de la prensión y la presión, sin dejar de observar unas buenas prácticas neuromotrices, visión y transcripción de izquierda a derecha, rotación habitual de los bucles y coger correctamente el instrumento u objeto de escritura (Ajuriaguerra, et al, 1973).

Respeto a la evolución del grafismo, Portellano (1989) nos habla de la fase preescriptora y la fase escritora. La primera incluye la evolución del niño desde los treinta y seis meses hasta los cinco años, encontrando primero un grafismo impulsivo, sin control, que poco a poco va madurando hasta que el niño ya tiene un control grafomotor. Este control le permite reproducir grafías elementales y al mismo tiempo se inicia la representación esquemática, hasta que finalmente, a los cuatro años, aparece el control de los movimientos grafomotrices que permitirá adquirir las primeras formas geométricas sencillas, para luego poder pasar a las más complejas, como sería dibujar un rombo cuando el niño ya tiene unos cinco años. Esta fase, la prescriptora, está relacionada con la etapa que se ha trabajado en esta propuesta, es decir, la etapa de Educación Infantil. La fase escritora, se inicia a partir de los cinco o seis años y da comienzo el aprendizaje sistemático de la escritura. Portellano afirma que gran parte de los fracasos escolares en los primeros cursos de la Educación Primaria se deben a un forzamiento de los procesos de maduración en niños que se inician en la escritura. Hasta convertirse en una actividad plenamente dinámica y fluida, debe pasar por las diferentes etapas enunciadas por Ajuriaguerra (1973), la fase precaligráfica, la fase caligráfica y la fase potscaligráfica. Si desde un principio el niño no tiene bien adquirido este grafismo o trazo, posteriormente pueden aparecer dificultades en la realización de la escritura. Conscientes ya de esta problemática, tanto Ajuriaguerra, Auzias, y Denner (1973) como Picq y Vayer (1977) propusieron programas para el abordaje reeducativo. Más recientemente, Pescar y Popescu (2012), propusieron un programa de desarrollo con un conjunto de ejercicios destinados a la prevención de la disgrafía y desórdenes de dislexia que proporcionó una inserción apropiada en la escuela.

Para que un niño logre la ejecución gráfica correcta, al iniciar el aprendizaje de la escritura, debe ser capaz de mantener su propio control postural, tener un dominio del brazo y una manera correcta de sostener el instrumento de escritura, así como orientarse en el espacio en el que debe escribir. Esto se puede conseguir, proporcionando a los niños una buena base psicomotriz, hecho que no siempre se presencia en la práctica escolar.
En la actualidad, Lica, Lizet, y González (2010) exploran la relación existente entre motricidad fina y las dificultades de escritura en un diseño de caso único en el que se evidenció la estrecha relación existente entre motricidad fina y escritura. Para adquirir una motricidad fina adecuada se requiere que el niño desarrolle las habilidades grafomotoras globales. Cisternas, Ceccato, Gil, y Marí Sanmillán (2014), indican que a los 4 años todas las variables neuropsicológicas examinadas presentan una correlación positiva con el conocimiento alfabético presentado por los niños, concluyendo que hay una fuerte relación entre las funciones neurocognitivas con las habilidades fundamentales y básicas a la hora de aprender a leer y escribir. González (2015), comparando la lectoescritura de dos grupos de primero de primaria de una escuela pública de la Ciudad de México, reporta mejoras importantes en la lectoescritura evaluada con la grafología del grupo de niños y niñas con el proceso de aprendizaje basado en la psicomotricidad.

Respecto a la relación a las dificultades en la psicomotricidad y el grafismo, López (2014) en su estudio detecta que casi un $40 \%$ de los alumnos de Primaria presentan una escritura deficiente, el $80 \%$ de los niños que han participado escriben mal porque no saben coger bien el lápiz y el $40 \%$ se coloca en mala posición cuando se pone a escribir. También entre los alumnos analizados, casi la mitad, ponen de forma incorrecta la hoja sobre la mesa o mueven mal la mano cuando avanzan mientras escriben. López, remarca que uno de los momentos clave para corregir una escritura deficiente es en primero de primaria, cuando los niños aprenden a utilizar la letra ligada. Según López, la corrección de la disgrafía es fundamental en el desarrollo académico de los estudiantes. En la misma línea, Guitart (2015), demuestra cómo pueden afectar los problemas de lateralidad en el rendimiento escolar.

\section{CONTEXTO}

Analizado el estado del arte y dada la importancia del proceso (incluso en las pruebas de Primaria del Departamento de Educación se mide la calidad del trazo), nos planteamos si el proceso de enseñanza aprendizaje que se sigue en algunos centros escolares es el adecuado. Se hace necesario dar más importancia al movimiento y la educación psicomotriz y utilizar las herramientas normativas que tenemos al alcance.

La finalidad principal del presente estudio es relacionar el desarrollo psicomotor con el grafismo y confirmar o no si a través de ejercicios de psicomotricidad fina y gruesa específicos en las extremidades superiores, siguiendo las leyes del desarrollo, se obtiene una mejora en el grafismo en alumnos de cuatro años.

La muestra estaba formada por 90 sujetos (37 niñas y 53 niños) de edades comprendidas entre los 4 y 5 años. De éstos, se seleccionaron 45 para el grupo experimental y otros 45 para el grupo control. Los sujetos procedían de dos escuelas diferentes de las Tierras del Ebro, una pública y una concertada. Se seleccionaron estas escuelas porque ambas ofrecen educación infantil y primaria y disponen de dos líneas por nivel educativo. En el caso de la escuela A, de los 39 sujetos matriculados en $\mathrm{P} 4$ se hicieron dos grupos aleatorios (pares / impares) quedando 20 sujetos en el grupo experimental y 19 en el grupo control. De los 20 sujetos del grupo experimental, se volvieron a dividir en dos grupos para poder trabajar por separado. En el caso de la escuela B, de los 
51 sujetos matriculados en el mismo curso se hicieron dos grupos aleatorios (pares / impares) quedando 25 sujetos en el grupo experimental y 26 en el grupo control. De los 25 sujetos del grupo experimental, se volvieron a dividir en dos grupos (12/13 sujetos) para poder trabajar por separado.

\section{DESCRIPCIÓN}

Se utilizó como instrumento de medida para la evaluación inicial y final el Método Dimensional Cambrodí (Cambrodí, Gràcia y Mercadé 1993). Este método está concebido y creado para evaluar sujetos comprendidos entre los 3 y 8 años. El contenido evaluado por el método se articula a través de cinco grandes campos de competencias o dimensiones, que pueden evaluarse de forma independiente. Concretamente, se utilizó la Dimensión Manipulativa y, dentro de ésta, el Sector IV: Organización perceptivo-manual aplicada al grafismo y el dibujo. Para realizar la evaluación, los resultados de los ítems se anotaron en unas hojas de registro para ver los resultados finales. Para cada ítem se otorgan las puntuaciones siguiendo los criterios de corrección. Puntuación: La escala de medida de esta prueba es de intervalos, puntuándose cada ítem entre 0 y 2 puntos, según la calidad de la respuesta. Este subtest consta de 20 ítems en orden de dificultad creciente desde copiar un círculo (ítem 1) hasta el manejo del compás (ítem 20); la mayoría de los cuáles exigen que el sujeto copie un modelo o una puntos para formar una imagen. De acuerdo con las tablas de baremo del manual, la puntuación habitual de los niños de entre 4 y 5 años, oscila entre los 8 y los 13 puntos.

El método cuantifica los resultados obtenidos mediante unos parámetros numéricos que permiten controlar matemáticamente los progresos, relacionar y comparar todos los valores y manipularlos estadísticamente.

El estudio consistió en un diseño cuasi experimental con medidas pre y pos-test con dos grupos, experimental y control. Se tomaron datos del grafismo antes y después de la intervención educativa. Previo a la iniciación del estudio se solicitó autorización a la dirección de los centros y a la familia por escrito para participar en el estudio. Los pasos seguidos para la aplicación de la presente investigación han sido la elección de la muestra y la asignación de los sujetos al grupo experimental y al grupo control, al azar; la aplicación del pretest en cada grupo sobre la variable dependiente (el grafismo); la intervención sólo al grupo experimental (12 sesiones de 15 minutos cada una), intentando que todas las condiciones fueran equivalentes en los dos grupos, de tal forma que la única diferencia fuese la influencia de la misma sesión.; y finalmente la aplicación del post-test sobre la variable dependiente a ambos grupos para concluir con el análisis de los resultados. Las pruebas fueron aplicadas individualmente a los 90 sujetos en horario escolar y las maestras y personal de recogida de datos recibieron tres horas de formación previas al inicio del estudio.

Para realizar la intervención al grupo experimental, se eligió un conjunto de actividades que trabajaran todos los movimientos del brazo, incluyendo de esta manera el hombro, el antebrazo, el codo, la muñeca, la mano y los dedos a través de la realización de ejercicios psicomotores implicados en el movimiento de estas partes del cuerpo, de esta manera también se siguieron las cuatro leyes del desarrollo y se procedió de acuerdo a la maduración armónica de estas partes. Estos ejercicios se dividieron en sesiones que se llevaron a cabo por

la mañana, en la franja horaria de 10 a 11 h, con una duración de 15 minutos. Estaban distribuidas en tres rincones de trabajo rotativos donde en cada uno se realizaban ejercicios de psicomotricidad gruesa y fina, para trabajar las extremidades superiores. En la elección de las actividades también se han tenido en consideración otros criterios con la finalidad que éstas fuesen fácilmente aplicables en la escuela. Presentamos unas actividades que son fáciles de ejecutar, se pueden realizar en la misma aula, no requieren profesorado especializado, son actividades que están inmersas en el juego y muchas de ellas son conocidas por los niños, no requieren un material específico, ni tampoco de alto coste.

Tabla 1. Actividades realizadas, descripción de las mismas y parámetro trabajado.

\begin{tabular}{|c|c|c|}
\hline Actividad & Descripción & Parámetro \\
\hline $\begin{array}{ll}1 . & \text { Bolos }\end{array}$ & $\begin{array}{l}\text { Consiste } \\
\text { derribar } 6 \text { bolos } \\
\text { con una pelota. }\end{array}$ & $\begin{array}{l}\text { En este juego se trabaja } \\
\text { la movilidad articular desde } \\
\text { el hombro hasta la mano y } \\
\text { la coordinación óculo- } \\
\text { manual. }\end{array}$ \\
\hline
\end{tabular}

2. Rodeo Los niños deben rodear con círculos los conos lanzándolos alrededor.

Se trabaja la movilidad del codo hasta la mano, la coordinación óculo-manual, la coordinación de los segmentos corporales y el control del tono muscular.

3. Pintores manuales

La actividad consiste en pintar un mural con la mano.

4. Pintores a pincel

\section{En}

actividad, se debe pintar un mural, pero en este caso con pincel. 5. Disco
volador

$\quad \begin{aligned} & \text { Se lanza } \\ & \text { disco }\end{aligned}$
horizontal
intentando
llegue lo más
posible.

6. Pásamela Este
consiste pasarse la pelota entre compañeros con la mano.

\begin{tabular}{ll} 
7. Bandejas & \multicolumn{1}{c}{ En esta } \\
de arena & actividad, cada \\
sujeto tiene una \\
bandeja de arena \\
$y$ ejecuta \\
diferentes dibujos \\
con el dedo.
\end{tabular}

8. Carretera Cada sujeto de coches tiene un coche $e$ intenta conducirlo por una carretera
Ésta se centra en el movimiento de todo el brazo y de la mano, la lateralidad y la coordinación óculo-manual.

Se potencia la motricidad fina, la pinza, el movimiento del codo y mano y el uso de un instrumento. También trabaja la lateralidad, la coordinación óculo-manual $\mathrm{y}$ el control del tono muscular de la mano.

Consiste en el movimiento de todo el brazo, desde el hombro hasta la mano, la lateralidad y la fuerza.

El trabajo se centra en el antebrazo y la mano, los flexores y extensores, la coordinación óculo-manual y el control muscular.

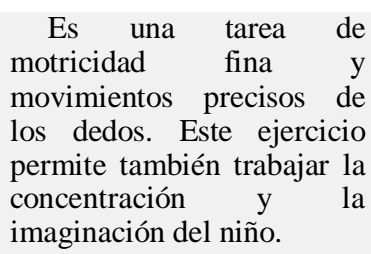
imaginación del niño.

Se trabaja el movimiento de la mano y la coordinación óculo-manual, 
dibujada en una la lateralidad, la tonicidad. alfombra.

Las sesiones se han distribuido, durante 6 semanas en el $2^{\circ}$ trimestre. Los alumnos del grupo experimental estaban organizados en tres rincones y en cada uno de ellos realizaban ejercicios de motricidad gruesa y fina de las extremidades superiores. Todos los alumnos pasaron por todos los rincones de juego y se producía el cambio de actividad cada cinco minutos.

Por último, tuvo lugar la evaluación final. Ésta se llevó a cabo una vez aplicadas las doce sesiones en cada escuela; se evaluaron de nuevo a los noventa sujetos individualmente, con el fin de observar si los cuarenta y cinco sujetos del grupo experimental, mostraban cambios respeto la evaluación inicial debidos a la intervención y verificar el proceso con el grupo control.

\section{Resultados}

Concluida la aplicación del programa de intervención, se procedió al análisis estadístico de las puntuaciones obtenidas por los sujetos en el pretest y postest.

En el grupo control, las puntuaciones del pretest oscilan entre 6 y 24 (rango= 18); en el postest oscilan entre 5 y 26 $($ rango $=21$ ). Respecto al grupo experimental, las puntuaciones del pretest oscilan entre 2 y 24 (rango= 22); en el postest, entre 2 y 33 (rango= 31). Se observa una gran variabilidad, mayor en el grupo experimental que en el grupo control, siendo ésta debida a los sujetos 11 y 29, en el grupo control, y a los sujetos 7 y 19, en el grupo experimental.

Un primer análisis comparativo pretende responder a dos cuestiones: la primera cuestión es si los dos grupos obtienen puntuaciones directas similares en el pretest; la segunda cuestión es si el grupo experimental obtiene un mayor rendimiento en el postest después de haber realizado las 12 sesiones de intervención propuestas.

Para comparar las puntuaciones obtenidas, se detallan a continuación los datos numéricos referentes a la media, mediana y desviación típica tanto del grupo control como del grupo experimental.

Tabla 2. Medias, medianas y desviaciones típicas de los grupos control y experimental.

\begin{tabular}{|l|c|c|c|}
\hline \multicolumn{3}{|c|}{ Estadística Grupo Control } \\
\hline Estad. & Pretest & Postest & Variación \\
\hline Media & 12,00 & 12,25 & 0,25 \\
Mediana & 13 & 13 & 0 \\
Desviación Típica & 3,03 & 3,06 & 0,03 \\
\hline
\end{tabular}

\begin{tabular}{|l|c|c|c|}
\hline \multicolumn{4}{|c|}{ Estadística Grupo Experimental } \\
\hline Estad. & Pretest & Postest & Variación \\
\hline Media & 10,46 & 14,93 & 4,47 \\
Mediana & 12 & 16 & 4 \\
Desviación Típica & 3,31 & 3,87 & 0,56 \\
\hline
\end{tabular}

Se observa que el grupo control sólo tiene una diferencia de 0,25 puntos entre el pretest y el postest. La mediana no varía y la desviación típica se sitúa en torno a 3 . En el grupo experimental, después de realizar el pretest y el postest, observamos una diferencia de 4,47 (pretest=10,46; postest $=14,93$ ). La mediana ha aumentado en 4 puntos y la desviación típica se sitúa en torno a los 4 puntos. Además, en el postest, tanto la puntuación media $(14,93)$ como la mediana (16), supera los valores esperados para este grupo de edad según el baremo del Método Dimensional Cambrodí. Destacamos que el grupo control, que sólo ha realizado actividades de papel y lápiz, no ha mejorado las puntuaciones (la media ha variado en 0,25 puntos y la mediana se mantiene igual).

A continuación, se expone de forma gráfica la interpretación de las puntuaciones medias (Figura 1) y de las puntuaciones medianas (Figura 2) antes y después del pre y postest, del grupo experimental y el grupo control.

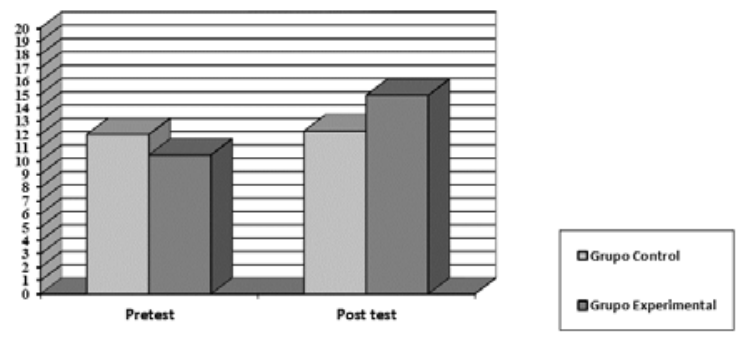

Figura 1. Comparación de las puntuaciones medias del grupo control y experimental.

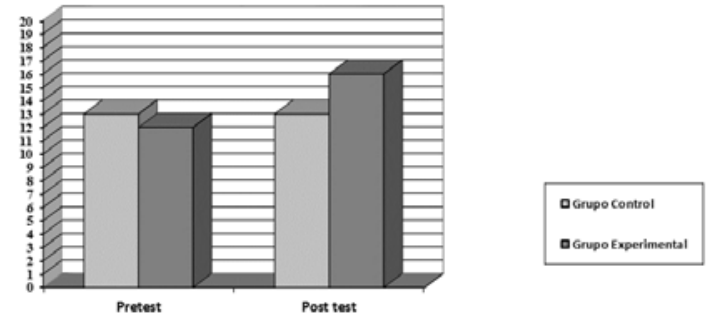

Figura 2. Comparación de las puntuaciones medianas del grupo control y experimental.

En las figuras se observa como el grupo control después de realizar el postest ha tenido muy poca variación. En cambio, en el grupo experimental, después de haber realizado la intervención durante doce sesiones, se observan unos resultados superiores. En el caso de la mediana, el grupo control no ha variado su mediana, se mantiene en 13 , mientras que en el grupo experimental la mediana ha pasado de 12 a 16.

Seguidamente, se plantea si estas diferencias en las puntuaciones obtenidas son significativas. Partimos de que la hipótesis nula (Ho) es que la variación de las puntuaciones entre del grupo experimental y el grupo control no diferirán significativamente después de haber efectuado la intervención y que la hipótesis alternativa (Ha) es que la variación de las puntuaciones del grupo experimental y el grupo control diferirán significativamente después de haber efectuado la intervención.

Para analizar los efectos que la intervención psicomotriz pudo tener en las variables estudiadas se efectuó un análisis 
estadístico utilizando el programa SPSS. Se utilizó la U de Mann Whitney como técnica de análisis de los datos obtenidos.

Tabla 3. Estadísticos descriptivos.

\begin{tabular}{|c|c|c|c|c|c|}
\hline & $\mathrm{N}$ & Media & D. típica & Mín. & Máx. \\
\hline Pretest & 90 & 11,69 & 3,224 & 2 & 24 \\
\hline Postest & 90 & 14,09 & 3,776 & 2 & 33 \\
\hline Grupo & 90 & 1,50 & 0,503 & 1 & 2 \\
\hline
\end{tabular}

Se observa que las medias y las desviaciones típicas son similares tanto en el pretest como en el postest. Se ofrecen también los rangos obtenidos. El rango promedio del grupo control ofrece una variabilidad media de 51,71 en el pretest y de 31,36 en el postest, mientras que el grupo experimental la variabilidad media es de 39,29 en el pretest y de 59,64 en el postest.

Efectuando la prueba U de Mann Whitney, obtenemos los siguientes estadísticos de contraste:

Tabla 4. Estadísticos de contraste.

\begin{tabular}{|l|l|}
\hline U de Mann-Whitney & 376,000 \\
W de Wilcoxon & 1411,000 \\
Z & $-5,184$ \\
Sig. Asintót. (bilateral) &, 000 \\
\hline
\end{tabular}

Como valor de la probabilidad $(p=0.000)<0.05$, debemos rechazar la hipótesis nula (Ho) y aceptar la hipótesis alternativa (Ha). En decir, con una confianza del 95\%, se evidencia que las puntuaciones obtenidas difieren significativamente entre las del grupo experimental y el grupo control. El grupo experimental ha obtenido unas puntuaciones superiores altamente significativas respecto al grupo control.

Podemos constatar a través del registro de calificaciones de los sujetos que la mayoría de miembros del grupo experimental han alcanzado una mejora en las copias de los dibujos después de la realización de las sesiones psicomotrices con actividades para trabajar las extremidades superiores (media pre-test $=10,46$ y post-test $=14,93$ ). Por ejemplo, en el pre-test, de los 45 sujetos del grupo experimental, 26 sujetos no sabían copiar un triángulo o lo hacían a medias $(57,7 \%)$ y en el post-test de estos 26, 13 lo hicieron correctamente (50\%), 11 de forma incompleta $(42,3 \%)$ y sólo dos obtuvieron 0 puntos (7,7\%). En cambio, de los 45 sujetos del grupo control, en el pre-test 24 de éstos no obtuvieron la máxima puntuación (53.3\%) y en el post-test 6 lo hicieron correctamente (25\%), 15 resultaron incompletos $(62,5 \%)$ y 3 sujetos no lo supieron copiar $(12,5 \%)$.

En conclusión, los resultados de este estudio ofrecen evidencia empírica sobre dos aspectos del aprendizaje grafomotor. En primer lugar, que los alumnos en los que el aprendizaje grafomotor se basa sólo en actividades de papel y lápiz no mejoran sus competencias; en segundo lugar, que los alumnos en los que el aprendizaje grafomotor se basa en actividades de papel y lápiz conjuntamente con actividades psicomotrices mejoran significativamente sus competencias.

\section{CONCLUSIONES}

Finalizado el estudio podemos dar respuesta a la hipótesis planteada, y tras el análisis de los resultados, podemos decir que la hipótesis se confirma. Los sujetos que han trabajado las extremidades superiores obtienen una mejora importante respeto a los sujetos que no han trabajado estas extremidades, $(p=0.000<0.05)$. Pescar y Popescu (2012), nos hablan de utilizar la psicomotricidad como carácter preventivo en su estudio y fruto de nuestra investigación consideramos primordial utilizarla no sólo como carácter preventivo si no de forma general en todo el grupo de alumnos.

En cuanto al objetivo propuesto en esta investigación (constatar si ejercitando las extremidades superiores, siguiendo las leyes del desarrollo, se obtiene una mejora en el grafismo), no ha sido hasta el final de la misma que se ha comprobado su cumplimiento. Aplicadas las doce sesiones de psicomotricidad en cada escuela, hemos comprobado que ejercitando las extremidades superiores, a través de actividades y siguiendo las leyes del desarrollo para favorecer un desarrollo armónico, se ha obtenido una mejora importante en los resultados de los niños, en relación al grafismo. Si bien no somos los primeros en proponer el aprendizaje de la grafomotricidad a partir de la reeducación psicomotriz (Ajuriaguerra, Auzias \& Denner, 1973, entre otros) hemos aplicado, diseñado y evaluado un planteamiento de utilización de la psicomotricidad como herramienta para mejorar la grafomotricidad.

Los resultados nos indican que la intervención ha funcionado y que haciendo ejercicios de psicomotricidad gruesa y fina ejercitando las extremidades superiores, podemos ayudar a mejorar el grafismo de los niños, hecho demostrado por los resultados de la presente investigación. En ella observamos que la mediana del grupo control no varía, se mantiene en 13 puntos y en cambio en el grupo experimental sí lo hace, pasando de 12 a 16.

Se ha comprobado que mediante la realización de unos ejercicios fáciles de aplicar, siguiendo el desarrollo madurativo y el desarrollo de las leyes del niño, ayudamos a mejorar los aprendizajes, en este caso el grafismo, y lo más importante es que estamos realizando psicomotricidad. Se han leído y analizado otros estudios, entre los que destacamos: González, 2015; Guitart, 2015; Cisternas, Ceccato, Gil, y Marí, 2014, que ponen de manifiesto que el hecho de no tener una psicomotricidad bien adquirida puede repercutir en problemas de aprendizaje de escritura, lectura y cálculo, de ahí la importancia de utilizar en esta investigación la psicomotricidad como base educativa y preventiva.

Los resultados del post-test del grupo experimental, nos confirman también la influencia directa del trabajo psicomotor en el grafismo, superando los valores esperados para este grupo de edad según el baremo del Método Dimensional Cambrodí.

Además de lo anterior, también debemos hacer referencia a la atención a la diversidad. Durante el trabajo de investigación nos hemos encontrado con tres datos atípicos de sujetos: uno de ellos presentaba necesidades educativas especiales y no ha mejorado, el otro sujeto presenta dificultades de aprendizaje y su puntuación respecto al pretest ha aumentado 6 puntos, que supone una media normal respeto el grupo control, y el último sujeto presenta capacidades de aprendizaje superiores y tanto 
en el pretest como el post-test sus puntuaciones destacan por encima del resto. Con ello, se pone de manifiesto que la psicomotricidad puede ayudar a los niños que presentan dificultades en ciertos aprendizajes, y un ejemplo de ello ha sido este caso. Así pues, esta investigación nos confirma la importancia de trabajar la psicomotricidad en los niños teniendo en cuenta su desarrollo madurativo, ya que muchas veces en las escuelas el proceso de enseñanza y aprendizaje de la escritura no tiene en cuenta que el niño aún no está suficientemente maduro o no está preparado para asumir un nuevo aprendizaje. Todo ello puede desencadenar dificultades en su adquisición y pérdida de interés, pudiéndonos llevar al fracaso escolar. De acuerdo con Viscarro (2014), la estructura actual del currículum de educación infantil otorga un rol importante al movimiento e integra y valora el trabajo psicomotor. El nuevo enfoque del currículum educativo facilita también la implementación de este tipo de dinámicas en el aula infantil y permite legalmente entender el grafismo desde la globalidad.

A partir de este estudio podemos deducir la necesidad de incluir, de forma habitual en las aulas, el trabajo de la motricidad gruesa y fina en la enseñanza - aprendizaje de las habilidades grafomotoras. Después de realizar esta investigación y de observar los resultados obtenidos, es necesario profundizar en determinados aspectos para próximas líneas de investigación sobre la psicomotricidad y el grafismo. Aunque la presente investigación sólo se ha llevado a cabo durante doce sesiones y los resultados han sido excelentes, sería conveniente proponer más sesiones para analizar los cambios a largo plazo, aumentar la muestra para poder obtener datos más generalizables e investigar en las distintas etapas del desarrollo (incluida la educación primaria) y comprobar si disminuyen los trastornos de escritura.

Este estudio sobre la psicomotricidad como recurso de mejora del grafismo, abre la puerta a nuevas investigaciones.

\section{REFERENCIAS}

Ajuriaguerra, J., Auzias, M., \& Denner, A. (1973a). La escritura del niño I. La evolución de la escritura y sus dificultades. Barcelona: Editorial Laia.

Albuquerque, A., \& Alves-Martins, M. (2016). Fomento de habilidades de lecto-escritura en la primera infancia. Estudio de seguimiento desde la educación infantil al primer curso de educación primaria. Infancia $y$ Aprendizaje: Journal for the Study of Education and Development 39, 3, 607-623. doi: 10.1080/02103702.2016.1196913

Alviz, G. (2012). La grafomotricidad en Educación Infantil. Revista Arista Digital. 16 (6), 48-54.

Auzias, M. (1978). Los trastornos de escritura infantil. Barcelona: Laia.

Calmy, G. (1977). La educación del gesto gráfico. Barcelona: Fontanella.

Cambrodí, A., Gràcia, J., \& Mercade, M. (1993). Método Dimensional Cambrodi. Exploración y valoración funcional del limitado mental. Tomo III-Dimensión “B”. Tarragona: Associación Tárraco Minusvàlidos.
Cisternas, Y., Ceccato, R., Gil, D., \& Marí Sanmillán, M.I. (2014). Neuropsychological functions in early literacy skills. International Journal of Developmental and Educational Psychology, 1 (1), 115-122. doi:10.17060/ijodaep.2014.n1.v1.354

Fonseca, V. (2008). Manual de observación psicomotriz. Barcelona: Inde Publicaciones, $3^{\text {a }}$.Edición.

González, M. (2015). La escritura en primer año de la escuela primaria y la psicomotricidad. Revista Psicomotricidad, Movimiento y Emoción, (1), p. 1-14.

Guitart, J. (2015, diciembre 29). ¿Cómo afectan los problemes de lateralidad en el rendimiento intel-lectual y emocional de los niños adolescentes y adultos?. La Vanguardia. Recuperado

de: http://www.lavanguardia.com/monograficos/diamundial-de-la-salud/como-afectan-los-problemas-delateralidad-en-el-rendimiento

Lica, M., Lizet, D., \& González, A. (2010). Relación entre ejecuciones deficientes de motricidad fina con dificultades de escritura: Análisi de un caso. Revista de Educación y Desarrollo, (13), p. 17-23.

López, A. (2014). La disgrafia a l'escola. +Grafo. Gabinet de Grafologia Aplicada. Recuperado de: http://www.mesgrafo.com/

Lurçat, L. (1988). Pintar, dibujar, escribir, pensar. El grafismo en el preescolar. Madrid: Cinel-Kapelusz.

Pescar, IT. \& Popescu, T. (2012). The importance of preschool education of psychomotricity component to prevent the instrumental disorders. Academia Science Journal. Psychologica Series, (1), p. 25-30.

Picq, L. \& Vayer, P. (1977). Educación Psicomotriz y retraso mental. Barcelona: Científico-Médica.

Portellano, J.A. (1985). La disgrafia. Madrid: CEPE.

Portellano, J.A. (1989). Fracaso escolar: diagnostico e intervención, una perspectiva neuropsicológica. Madrid: CEPE

Shatil, E., Share, D. L., \& Levin, I. (2000). On the contribution of kindergarten writing to grade 1literacy: A longitudinal study in Hebrew. Applied Psycholinguistics, 21, 1-21. doi:10.1017/S0142716400001016

Sugrañes, E., Àngel, M., Andrés, J., Colomé, M.T., Martí, R. M., Martín, M., \& Pinell, N. (...) (2007). La educación psicomotriz (3-8 años). Cuerpo, movimiento, percepción, afectividad: una propuesta teórico-práctica. Barcelona: Graó.

Tolchinsky, L. (2005). The emergence of writing. In C. MacArthur, S. Graham, \& J.Fitzgerald (Eds.), Handbook of writing research (pp. 83-96). New York, NY:Guilford.

Viscarro, I. (2014). Tema d’anàlisi: Quina és la situació actual de l'educació psicomotriu?, quines són les perspectives de futur d'aquesta disciplina? Revista de Ciències de l'Educació, p. 99-106. 